\title{
ERROR AND INFERENCE: AN OUTSIDER STAND ON A FREQUENTIST PHILOSOPHY
}

\author{
CHRISTIAN P. ROBERT \\ UNIVERSITÉ PARIS-DAUPHINE \\ CEREMADE, IUF, AND CREST, \\ 75775 PARIS CEDEX 16, FRANCE \\ XIAN@CEREMADE.DAUPHINE.FR
}

\begin{abstract}
This note is an extended review of the book Error and Inference, edited by Deborah Mayo and Aris Spanos, about their frequentist and philosophical perspective on testing of hypothesis and on the criticisms of alternatives like the Bayesian approach.
\end{abstract}

\section{Introduction.}

"The philosophy of science offer valuable tools for understanding and advancing solutions to the problems of evidence and inference in practice"D. Mayo and A. Spanos, p.xiv, Error and Inference, 2010

This philosophy book, Error and Inference, whose subtitle is "Recent exchanges on experimental reasoning, reliability, and the objectivity and rationality of Science", includes contributions by P. Achinstein, A. Chalmers, D. Cox, C. Glymour, L. Laudan, A. Musgrave, and J. Worrall, and by both editors, D. Mayo and A. Spanos. It offers reflections on the nature of testing and the defence of the "Error-Statistical philosophy", proposed by Mavo (1996). Error and Inference is the edited continuation of a conference held at Virginia Tech in 2006, ERROR 06. Given my layman interest in the philosophy of Science, I find that the book reads rather well, despite my missing references in the field. (Paradoxically, the sections I found the hardest to follow were those most centred on Statistics.) The volume is very carefully edited and thus much more than a collection of papers, thanks to the dedications of the editors. In particular, Deborah Mayo added her comments at the end of the chapters of all contributors (but her own's and Aris Spanos'). Her strongly frequentist perspective on the issues of testing and model choice are thus reflected in the tone of the volume, even though some contributors bring some level of (un-Bayesian) contradiction to the debate. My own Bayesian arguments following from reading Error and Inference are provided in the following sections, ordered by book chapters. A broader and deeper philosophico-statistical perspective on the nature of Bayesian inference to which I mostly subscribe are given by Berger (2003) and Gelman and Shalizi (2012).

\section{Severe testing.}

"However, scientists wish to resist relativistic, fuzzy, or post-modern turns (...) Notably, the Popperian requirement that our theories are testable and falsifiable is widely regarded to contain important insights about responsible science and objectivity."-D. Mayo and A. Spanos, p.2, Error and Inference, 2010

As illustrated by the above quote (which first part I obviously endorse), the overall perspective in the book is Popperian, despite Popper's criticism of statistical inference as a whole and of Bayesian statistics as a particular (see the quote at the top of Section 5.1). Another fundamental concept throughout the book is the "ErrorStatistical philosophy" whose Deborah Mayo is the proponent (Mayo, 1996). One of the tenets of this philosophy is a reliance on statistical significance tests in the fusion Fisher-Neyman-Pearson (or self-labelled frequentist) tradition, along with a severity 
principle ("We want hypotheses that will allow for stringent testing so that if they pass we have evidence of a genuine experimental effect", p.19) stated as (p.22)

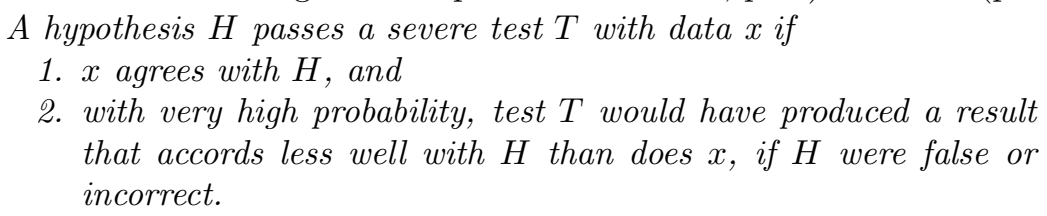

The $p$-value is advanced as a direct accomplishment of this goal, but I fail to see why it does or, if it does, then why a Bayes factor (Jeffreys, 1939) would not. Indeed, the criterion depends on the definition of the probability model when $H$ is false or incorrect. This somehow reflects negatively Mayo's criticism of the Bayesian approach, as explained below. In particular, the "high probability" assumes a lack of contiguity between the tested hypothesis $H$ and its negative $\neg H$, that is unrealistic even within the perspective of the book, as is the claim below. (See also Spanos' required "highly improbable" good fit to the model defined by $H$ "were $H$ to be in error", p.209.)

"Formal error-statistical tests provide tools to ensure that errors will be correctly detected with high probabilities." — D. Mayo, p.33, Error and Inference, 2010

In Chapter 1 (Learning from error, severe testing, and the growth of theoretical knowledge), Deborah Mayo address a frontal attack against the Bayesian approach. The main criticism therein is about the Bayesian approach to testing (restrictedly defined through the posterior probability of the hypothesis, rather than through the predictive) is about the "catchall hypothesis", a somehow desultory term replacing the more standard "alternative" hypothesis. According to Mayo, this alternative hypothesis should "include all possible rivals, including those not even though of" (p.37). This sounds to me like a weak argument, given that

1. the argument should also apply in the frequentist case, in order to define the relevant probability distribution "when $H$ is false or incorrect" (see, e.g., the above quote and also the one about the "probability of so good an agreement (between $H$ and $x$ ) calculated under the assumption that $H$ is false", p.40);

2. it is reasonable to argue that a well-defined alternative should always be available given testing an hypothesis is very rarely the ultimate goal of a study: if $H$ is rejected, there should be a available alternative model for conducting the analysis of the available data, to be picked or constructed precisely among those "thought of";

3. the argument about the infinite set of possible rivals is self-defeating in that it leads to the fallacy of the perfect model: given a dataset, there always is a model that fits perfectly this dataset;

4. rejecting or accepting an hypothesis $H$ in terms of the sole null hypothesis $H$ does not make sense from operational as well as from game-theoretic (DeGroot, 1970) perspectives.

A further argument found in this chapter that the posterior probability of $H$ is a direct function of the prior probability of $H$ does not stand when used against the Bayes factor. (The same lack of justification applies to the criticism that the Bayesian approach does not accommodate newcomers, i.e., new alternatives, since marginal likelihoods are computed separately for each potential model, Kass and Raftery, 1995.) Stating that "one cannot vouch for the reliability of [this Bayesian] procedure - that it would rarely affirm theory $T$ were $T$ false" (p.37) completely ignores the wealth of results about the consistency of the Bayes factor (Schervish, 1995, Berger et al., 2003, 
Moreno et al., 2010), since the "asymptotic long run" (p.20) matters in the ErrorStatistical philosophy. The final argument that Bayesians rank "theories that fit the data equally well (i.e., have identical likelihoods)" (p.38) does not account for-or dismisses, see page 50 referring to Jeffreys and Berger instead of Berger and Jeffervs (1992) - the fact that Bayes factors are automated Occam's razors in that the averaging of the likelihoods over spaces of different dimensions are natural advocates of simpler models (MacKay, 2002). Even though I discuss this issue in the following section, Mayo also seems to imply there that Bayesians are using the data twice (this is at least how I interpret the insistence on "same" p. 50), which is a sin [genuine] Bayesian analysis can hardly be found guilty of! Overall, the undercurrents of those criticisms is similar to the ones found in the recent attacks of Templeton (2008, 2010), later rebuked in, e.g., Beaumont et al. (2010), about apparently the approximative Bayesian calculation methods but in truth directed to the overall Bayesian handling of testing of hypotheses.

\section{Theory confirmation and model evidence (Chapter 4).}

"Taking the simple case where the background principles specify a finite list of alternatives $T_{1}, \ldots, T_{n}$, each piece of data falsifies some $T_{i}$ until we are left with just one theory $T_{j}$-which (...) is thus 'deduced from the phenomenon'.'" - J. Worrall, p.134, Error and Inference, 2010

The fourth chapter of Error and Inference, written by John Worrall, covers the highly interesting issue of "using the data twice". The point has been debated many times in the Bayesian literature and this is one of the main criticisms raised against Aitkin's (2010) integrated likelihood. Worrall's perspective is both related and unrelated to this purely statistical issue, when he considers that "you can't use the same fact twice, once in the construction of a theory and then again in its support" (p.129). (He even signed a "UN Charter", where UN stands for "use novelty"!) After reading both Worrall's and Mayo's viewpoints, the later being that all that matters is severe testing as it encompasses the UN perspective, I afraid I am none the wiser about the relevance of their arguments from a statistical perspective, but this led me to reflect on the statistical issue 1

From first principles, a Bayesian approach should only use the data once, namely when constructing the posterior distribution on every unknown component of the model(s). Given this all-encompassing posterior, all inferential aspects are the consequences of a sequence of decision-theoretic steps in order to select optimal procedures. This is the ideal setting while, in practice, relying on a sequence of posterior distributions is often necessary, each posterior being a consequence of earlier decisions, which makes it the result of a multiple use of the data... For instance, the process of Bayesian variable selection is on principle clean from the sin of "using the data twice": one simply computes the posterior probability of each of the variable subsets and this is over. However, in a case involving many (many) variables, there are two difficulties: one is about building the prior distributions for all possible models, a task that needs to be automatised to some extent; another is about exploring the set of potential models, e.g., resorting to projection priors as in the intrinsic solution of Pérez and Berger (2002), while unavoidable and a "least worst" solution, means switching priors/posteriors based on earlier acceptances/rejections, i.e. on the data.

\footnotetext{
${ }^{1}$ However, the UN principle seems much too mechanical when considering the quote from John Worrall: ticking models away by using one observation at a time is certainly not a sound statistical procedure, even though sufficient and testing statistics may vary from one model to the next (Robert et al., 2011).
} 
Second, the path of models truly explored [which will be a minuscule subset of the set of all models] will depend on the models rejected so far, either when relying on a stepwise exploration or when using a random walk MCMC algorithm. Although this is not crystal clear (there actually is plenty of room for arguing the opposite!), it could be argued that the data is thus used several times in this process...

"Although the [data] set as a whole both fixes parameter values and (unconditionally) supports, no particular element of the data set does both."J. Worrall, p.140, Error and Inference, 2010

One paragraph in Worrall's chapter intersects with the previous discussion, while getting away from the "using the data twice" discussion. It compares two theories with a different number of "free" parameters, hence (seemingly) getting different amounts of support from a given dataset (" $n$ lots of confirmation [versus] $n-r$ lots", p.140). The distinction sounds too arithmetic in that the algebraic dimension of a parameter may be only indirectly related to its information dimension, as illustrated by DIC (Spiegelhalter et al., 2002), although this criterion does indeed use the data twice. Furthermore, a notion like the fractional Bayes factor (O'Hagan, 1995) shows that the whole dataset may contribute both to the model selection and to the parameter estimation without the above dichotomy to occur.

\section{Theory testing in economics and the error-statistical perspective (Chapter 6). \\ "Statistical knowledge is independent of high-level theories." — A. Spanos, p.242, Error and Inference, 2010}

The sixth chapter of Error and Inference is written by Aris Spanos and deals with the issues of testing in econometrics (rather than economics). It provides on the one hand a fairly interesting entry in the history of economics and the resistance to databacked theories, primarily because the buffers between data and theory are multifold ("huge gap between economic theories and the available observational data", p.203). On the other hand, what I fail to understand in the chapter (and in other parts of Error and Inference) is the (local) meaning of theory, as it seems very distinct from what I would call a (statistical) model. The sentence "statistical knowledge, stemming from a statistically adequate model allows data to 'have a voice of its own's (...) separate from the theory under scrutiny and its succeeds in securing the frequentist goal of objectivity in theory testing" (p.206) is puzzling in this respect. (Actually, I would have liked to see a clear meaning put to this "voice of its own", as it otherwise sounds mostly as a catchy sentence...) Similarly, Spanos distinguishes between three types of models: primary/theoretical, experimental/structural: "the structural model contains a theory's substantive subject matter information in light of the available data" (p.213), data/statistical: "the statistical model is built exclusively using the information contained in the data" (p.213). I have trouble to understand how testing can distinguish between those types of models: as a naïve reader, I would have thought that only the statistical model could be tested by a statistical procedure, even though I would not call the above a proper definition of a statistical model (esp. since Spanos writes a few lines below that the statistical model "would embed (nest) the structural model in its context" (p.213)). The normal example followed on pages 213-217 does not help [me] to put meaning on this distinction: it simply illustrates the impact of failing some of the defining assumptions (normality, time homogeneity [in mean and variance], independence). (As an aside, the discussion about the poor estimation of the correlation p.214-215 does not help, because it involves a second variable $Y$ that is not defined for this example.) It would be nice of course if the "noise" in 
a statistical/econometric model could be studied in complete separation from the structure of this model, however they seem to be irremediably intermingled to prevent this partition of roles. I thus do not see how the "statistically adequate model is independent from the substantive information" (p.217), i.e. by which rigorous process one can isolate the "chance" parts of the data to build and validate a statistical model per se. The simultaneous equation model (SEM, pp.230-231) is more illuminating of the distinction set by Spanos between structural and statistical models/parameters, even though the difference in this case boils down to a question of identifiability.

"What is needed is a methodology of error inquiry that encourages detection and identification of the different ways an inductive inference could be in error by applying effective procedures that would detect such errors when present with very high probability." - A. Spanos, p.241, Error and Inference, 2010

The chapter, in line with the book, is strongly entrenched within the "F-N-P" frequentist paradigm" (p.210). Obviously, there are major differences between the Fisherian and the Neyman-Pearson approaches to testing that are not addressed in the chapter, the main opposition being the role (or non-role) of the $p$-value. The recurrent (and relevant) worry of Spanos about model misspecification is not directly addressed by either of those. The extremely strong criticisms of "cookbook" econometrics textbooks (p.233) could thus be equally addressed to most statistics books and papers: I do not see how the "error statistical perspective" could be able to spot all departures from model assumptions. Section 6.3 comparing covariate dependent models à la Cowles Commission with standard autoregressive models is thus puzzling because (a) they do not seem particularly comparable to me, for the very reason evacuated by Spanos that "ARIMA models ignore all substantive information", and (b) time series models may be just as well misspecified. To think that adding a linear time-dependence to a regression model is sufficient to solve the issues, as argued by Spanos ("...what distinguishes [this] approach from other more data-oriented traditions is its persistent emphasis on justifying the methodological foundations of its procedures using the scientific credentials of frequentist inference", p.238), is a rather radical shortcut for a justification of the approach.

\section{New perspectives on (some old) problems of frequentist statistics (Chapter 7). \\ "The defining feature of an inductive inference is that the premises (evi- dence statements) can be true while the conclusion inferred may be false without a logical contradiction: the conclusion is "evidence transcending"." \\ - D. Mayo and D. Cox, p.249, Error and Inference, 2010}

The seventh chapter of Error and Inference is divided in four parts, written by David Cox, Deborah Mayo, and Aris Spanos, in different orders and groups of authors. This is certainly the most statistical of all chapters, not a surprise when considering that David Cox was involved. Overall, this chapter is crucial by its contribution to the debate on the nature of statistical testing.

\subsection{Frequentist statistics as a theory of inductive inference (Part I).}

"The advantage in the modern statistical framework is that the probabilities arise from defining a probability model to represent the phenomenon of interest. Had Popper made use of the statistical testing ideas being developed at around the same time, he might have been able to substantiate his account of falsification." - D. Mayo and D. Cox, p.251, Error and Inference, 2010 
The first part of the chapter is Mayo's and Cox theory of inductive inference. It was first published in the 2006 Erich Lehmann symposium (Mayo and Cox, 2006). Contrary to Part II, there is absolutely no attempt there to link nor clash with the Bayesian approach: this part is focussing on frequentist statistical theory as the sole basis for inductive inference. The debate therein about deducing that $H$ is correct from a dataset successfully facing a statistical test is classical (in both senses) but I stand unmoved by the arguments. The null hypothesis $H$ remains the calibrating distribution throughout the chapter, with very little (or at least not enough) consideration of what happens when the null hypothesis does not hold. Section 3.6 [of this part] about confidence intervals being another facet of testing hypotheses is representative of this perspective. The $p$-value is defended as the central tool for conducting hypothesis assessment. (In this version of the paper, some $p$ 's are written in roman characters and others in italics, which is a wee confusing until one realises that this is a mere typo!) The fundamental imbalance problem, namely that, in contiguous hypotheses, a test cannot be expected both to most often reject the null $H$ when it is [very moderately] false and to most often accept the null $H$ when it is correct is not discussed there. As stated in the introduction, the argument about substantive nulls found in Section 3.5 applies to a stylised case of well-separated scientific theories, however the real world of models is more similar to a grayish (and more Popperian?) continuum of possibles. In connection with this, I would have expected that the book addressed on philosophical grounds George Box's aphorism that "all models are wrong". Indeed, one (both philosophical and methodological) difficulty with both the $p$-values and the frequentist evidence principle (FEV) is that they rely on the strongly restrictive belief that one given model can be exact or true (while criticising the subjectivity of the prior modelling in the Bayesian approach). Even in the typology of types of null hypotheses drawn by the authors in Section 3, the "possibility of model misspecification" is addressed in terms of the low power of an omnibus test, while agreeing that "an incomplete probability specification" is unavoidable. An argument found at several place in Error and Inference is that the alternative to $H$ cannot be completely specified, an argument going against the second part of Box's aphorism that "some models are more useful than others".

"Sometimes we can find evidence for $H_{0}$, understood as an assertion that a particular discrepancy, flaw, or error is absent, and we can do this by means of tests that, with high probability, would have reported a discrepancy had one been present." - D. Mayo and D. Cox, p.255, Error and Inference, 2010

The above quote relates to the Failure and Confirmation section where the authors try to push the argument in favour of frequentist tests one step further, namely that that "moderate $p$-values" may sometimes be used as confirmation of the null. (I may have misunderstood, the end of the section appearing as a defence of a purely frequentist - as in repeated experiments - interpretation. This reproduces an earlier argument about the nature of probability found in Section 1.2, as characterising the "stability of relative frequencies of results of repeated trials".)

As an aside, this chapter made me ponder afresh about the nature of probability, a debate that put me off so much in Keynes (1920) and even in Jeffreys (1939) (see Robert et al., 2009, Robert, 2010). From a mathematical formal perspective, there is only one "kind" of probability, the one defined via a reference measure and a probability, whether it applies to observations or to parameters. From a philosophical perspective, there is a natural issue about the "truth" or "realism" of the probability 
quantities and of the probabilistic statements. Error and Inference and in particular this chapter consider that a truthful probability statement is the one agreeing with "a hypothetical long-run of repeated sampling, an error probability", while the statistical inference school of Keynes (1921), Jeffreys (1939), and Carnap (1952) "involves quantifying a degree of support or confirmation in claims or hypotheses", which makes this (Bayesian) approach sound less realistic. Obviously, I have no ambition to solve this long-standing debate, however I see no reason in the first approach to be more realistic by being grounded on stable relative frequencies à la von Mises (1957). If nothing else, the notion that a test should be evaluated on its long run performances is very idealised as the concept relies on an ever-repeating and infinite sequence of identical trials. Relying on probability measures as self-coherent mathematical measures of uncertainty carries (for me) as much (or as little) reality as the above infinite experiment. Now, the paper is not completely entrenched in this interpretation, e.g. when it concludes that "what makes the kind of hypothetical reasoning relevant to the case at hand is not the long-run low error rates associated with using the tool (or test) in this manner; it is rather what those error rates reveal about the data generating source or phenomenon" (p.273).

\begin{abstract}
"If the data are so extensive that accordance with the null hypothesis implies the absence of an effect of practical importance, and a reasonably high $p$ value is achieved, then it may be taken as evidence of the absence of an effect of practical importance."- D. Mayo and D. Cox, p.263, Error and Inference, 2010
\end{abstract}

The paper discusses on several occurrences conclusions to be drawn from a $p$ value near one, as in the above quote. This is an interpretation that does not sit well with my (and others', e.g. Hwang et al., 1992) understanding of $p$-values being distributed as uniforms under the null: very high $p$-values should be as suspicious as very low $p$-values. (The criticism is not new, of course, see Jeffrevs, 1939.) Unless one does not strictly adhere to the null model, which brings back the above issue of the approximativeness of any model. I also found fascinating to read the criticism that "power appertains to a prespecified rejection region, not to the specific data under analysis" as I thought this equally applied to the $p$-values, turning "the specific data under analysis" into a departure event of a prespecified kind. (And leading to the famous aphorism by Jeffreys, 1939, about an "hypothesis that may be true may be rejected because it has not predicted observable results that have not occurred".)

\title{
5.2. Objectivity and conditionality in frequentist inference (Part II).
}

"Frequentist methods achieve an objective connection to hypotheses about the data-generating process by being constrained and calibrated by the method's error probabilities in relation to these models ."-D. Cox and D. Mayo, p.277, Error and Inference, 2010

The second part of the seventh chapter of Error and Inference, is due to David Cox and Deborah Mayo. The purpose is clear and the sub-chapter quite readable from a statistician's perspective. I however find it difficult to quantify objectivity by first conditioning on "a statistical model postulated to have generated data", as again this assumes the existence of a "true" probability model where "probabilities (...) are equal or close to the actual relative frequencies". As earlier stressed by Andrew Gelman on his blog,

"I don't think it's helpful to speak of "objective priors." As a scientist, I try to be objective as much as possible, but I think the objectivity comes in the principle, not the prior itself. A prior distribution-any statistical 
model-reflects information, and the appropriate objective procedure will depend on what information you have."Andrew Gelman

This part opposes the likelihood, Bayesian, and frequentist methods, as in what Gigerenzer (2002) classifies as the "superego, the ego, and the id". Cox and Mayo stress from the start that the frequentist approach is (more) objective because it is based on the sampling distribution of the test. My primary problem with this thesis is that the "hypothetical long run" (p.282) does not hold in realistic settings. Even in the event of a reproduction of similar or identical tests, a sequential procedure exploiting everything that has been observed so far is more efficient than the mere replication of the same procedure solely based on the current observation.

"Virtually all (...) models are to some extent provisional, which is precisely what is expected in the building up of knowledge."-D. Cox and D. Mayo, p.283, Error and Inference, 2010

The above quote is something I completely agree with, being another phrasing of George Box's "all models are wrong", but this transience of working models is a good reason in my opinion to account for the possibility of alternative working models from the start of the statistical analysis. Hence for an inclusion of those models in the statistical analysis equally from the start. Which leads almost inevitably to a Bayesian formulation of the testing problem.

"Perhaps the confusion [over the role of sufficient statistics] stems in part because the various inference schools accept the broad, but not the detailed, implications of sufficiency."-D. Cox and D. Mayo, p.286, Error and Inference, 2010

The discussion over the sufficiency principle is quite interesting, as always. The authors propose to solve the confusing opposition between the sufficiency principle and the frequentist approach by assuming that inference "is relative to the particular experiment, the type of inference, and the overall statistical approach" (p.287). This constraint creates a barrier between sampling distributions that avoids the "binomial versus negative binomial" paradox often advanced in the Bayesian literature (Berger and Wolpert, 1988). But the authors' solution is somehow tautological: by conditioning on the sampling distribution, it avoids the difficulties linked with several sampling distributions all producing the same likelihood 2 The section (pp.288-289) is also revealing about the above "objectivity" of the frequentist approach in that the derivation of a test taking large value away from the null with a well-known distribution under the null is not an automated process, esp. when nuisance parameters cannot be escaped from (pp.291-294). Achieving separation from nuisance parameters, i.e. finding statistics that can be conditioned upon to eliminate those nuisance parameters, does not seem feasible outside well-formalised models related with exponential families. Even in such formalised models, a (clear?) element of arbitrariness is involved in the construction of the separations, which implies that the objectivity is under clear threat. The chapter recognises this limitation in Section 9.2 (pp.293-294), however it argues that separation is much more common in the asymptotic sense and opposes the approach to the Bayesian averaging over the nuisance parameters, which "may be vitiated by faulty priors" (p.294). I am not convinced by the argument, given that the (approximate) condition approach amount to replace the unknown nuisance

\footnotetext{
${ }^{2}$ From a practical perspective, I have however become less enamoured of the sufficiency principle as the existence of [non-trivial] sufficient statistics is quite the rare event. Especially across models, as discussed in Robert et al. (2011).
} 
parameter by an estimator, without accounting for the variability of this estimator. Averaging brings the right (in a consistency sense) penalty.

A compelling section is the one about the weak conditionality principle (pp.294298), as it objects to the usual statement that a frequency approach breaks this principle. In a mixture experiment about the same parameter $\theta$, inferences made conditional on the experiment "are appropriately drawn in terms of the sampling behaviour in the experiment known to have been performed" (p. 296). This seems hardly objectionable, as stated. And I must confess the sin of stating the opposite as The Bayesian Choice has this remark (Robert (2007), Example 1.3.7, p.18) that the classical confidence interval averages over the experiments. The term experiment validates the above conditioning in that several experiments could be used to measure $\theta$, each with a different $p$-value. I will not argue with this: I could, however, about "conditioning is warranted to achieve objective frequentist goals" (p. 298) in that the choice of the conditioning, among other things, weakens the objectivity of the analysis. In a sense the above pirouette out of the conditioning principle paradox suffers from the same weakness, namely that when two distributions characterise the same data (the mixture and the conditional distributions), there is a choice to be made between "good" and "bad". Nonetheless, an approach based on the mixture remains frequentist if non-optimal. (The chapter later attacks the derivation of the likelihood principle by Birnbaum, 1962 in Part III by Deborah Mayo that will not be discussed in this paper as it deserves a specific paper.)

\section{"Many seem to regard reference Bayesian theory to be a resting point until satisfactory subjective or informative priors are available. It is hard to see how this gives strong support to the reference prior research program."- D. Cox and D. Mayo, p.302, Error and Inference, 2010}

A section also worth commenting is (unsurprisingly!) the one addressing the limitations of the Bayesian alternatives (pp.298-302). The authors however dismiss straight away the personalistic approach to prior construction by considering it fails the objectivity canons. This seems too hasty to me, since the choice of a prior is

1. the choice of a reference probability measure against which to assess the information brought by the data, not clearly less objective than picking one frequentist estimator or another, and

2. a personal construction of the prior can also be defended on objective grounds, based on the past experience of the modeller. That it varies from one modeller to the next is not an indication of subjectivity per se, simply of different past experiences.

Cox and Mayo then focus on reference priors, à la Bernardo-Berger (1992), once again pointing out the lack of uniqueness of those priors as a major flaw. While the sub-chapter agrees on the understanding of those priors as convention or reference priors, aiming at maximising the information input from the data, it gets stuck on the impropriety of such priors: "if priors are not probabilities, what then is the interpretation of a posterior?" (p.299). This seems like a strange (and unfair) comment to me: the interpretation of a posterior is that it is a probability distribution and this is the only mathematical constraint one has to impose on a prior. (Which may indeed be a problem in the derivation of reference priors.) As detailed in The Bayesian Choice among other books (Hartigan, 1983, Berger, 1985), there are many compelling reasons to invite improper priors into the game. (And one not to, namely the difficulty with point null hypotheses.) While I agree that the fact that some reference priors (like matching priors, whose discussion p.302 escapes me) have good frequentist properties 
is not compelling within a Bayesian framework, it seems a good enough answer to the more general criticism about the lack of objectivity: in that sense, frequency-validated reference priors are part of the huge package of frequentist procedures and cannot be dismissed on the basis of being Bayesian. That reference priors are possibly at odd with the likelihood principle does not matter so much: the shape of the sampling distribution is part of the prior information, not of the likelihood per se. The final argument (Section 12) that Bayesian model choice requires the preliminary derivation of "the possible departures that might arise" (p.302) has been made at several points in Error and Inference and already discussed above. Besides being in my opinion a valid working principle, i.e. selecting the most appropriate albeit false model, this definition of well-defined alternatives is mimicked by the assumption of "statistics whose distribution does not depend on the model assumption" (p.302) found in the same last paragraph.

In conclusion this (sub-)chapter by David Cox and Deborah Mayo is (as could be expected!) a deep and thorough treatment of the frequentist approach to the sufficiency and (weak) conditionality principle. It however fails to convince me that there exists a "unique and unambiguous" frequentist approach to all but the most simple problems. At least, from reading this chapter, I cannot find a working principle that would lead me to this single unambiguous frequentist procedure.

\subsection{Spanos' comments (Part IV).}

"It is refreshing to see Cox and Mayo give a hard-nosed statement of what scientific objectivity demands of an account of statistics, show how it relates to frequentist statistics, and contrast that with the notion of "objectivity" used by O-Bayesians."-A. Spanos, p.326, Error and Inference, 2010

The discussion by Aris Spanos is an exegesis of Part II by David Cox and Deborah Mayo: the first point in the discussion is that the above is "a harmonious blend of the Fisherian and N-P perspectives to weave a coherent frequentist inductive reasoning anchored firmly on error probabilities"(p.316). The discussion by Spanos being very much a-critical, I will rather expose here some thoughts of mine that came from reading this apology. (Remarks about Bayesian inference are limited to some piques like the above, which only reiterates those found earlier [and later: "the various examples Bayesians employ to make their case involve some kind of "rigging" of the statistical model", Aris Spanos, p.325; "The Bayesian epistemology literature is filled with shadows and illusions", Clark Glymour, p. 335] in Error and Inference.)

The "general frequentist principle for inductive reasoning" (p.319) at the core of Cox and Mayo's paper is obviously the central role of the $p$-value in "providing (strong) evidence against the null $H_{0}$ (for a discrepancy from $H_{0}$ )". Once again, I fail to see it as the epitome of a working principle in that

1. it depends on the choice of a divergence measure $d(z)$, which reduces the information brought by the data $z$;

2. it does not articulate the level for labelling nor the consequences of finding a low $p$-value;

3. it ignores the role of the alternative hypothesis.

Furthermore, Spanos' discussion deals with "the fallacy of rejection" (pp.319-320) in a rather artificial (if common throughout Error and Inference) way, namely by setting a buffer of discrepancy around the null hypothesis. While the choice of a maximal degree of precision sounds natural to me (in the sense that a given sample size should not allow for the discrimination between two arbitrary close values of the parameter), the fact that $\gamma$ is in fine set by the data (so that the $p$-value is high) is fairly puzzling. 
If I do understand correctly, the change from a $p$-value to a discrepancy $\gamma$ is a fine device to make the "distance" from the null better understood, but it has an extremely limited range of application. If I do not understand correctly, the discrepancy $\gamma$ is fixed by the statistician and then this sounds like an extreme form of prior selection.

There is another issue I do not understand in this part, namely the meaning of the severity evaluation probability

$$
P\left(d(Z)>d\left(z_{0}\right) ; \mu>\mu_{1}\right)
$$

as the conditioning on the range of parameter values seems impossible in a frequentist setting. This leads me to an idle and unrelated questioning as to whether there is a solution to

$$
\sup _{d} \mathbb{P}_{H_{0}}\left(d(Z) \geq d\left(z_{0}\right)\right)
$$

as this would be the ultimate discrepancy $\gamma$. Or whether this does not make any sense, because of the ambiguous role of $z_{0}$, which somehow needs to be integrated out. (Otherwise, $d$ can be chosen so that the probability is 1.)

"If one renounces the likelihood, the stopping rule, and the coherence principles, marginalizes the use of prior information as largely untrustworthy, and seek procedures with 'good' error probabilistic properties (whatever that means), what is left to render the inference Bayesian, apart from a belief (misguided in my view) that the only way to provide an evidential account of inference is to attach probabilities to hypotheses?"-A. Spanos, p.326, Error and Inference, 2010

The role of conditioning on ancillary statistics is emphasized both in the paper and in the discussion. This conditioning clearly reduces variability, however there is no reservation about the arbitrariness of such ancillary statistics. And the fact that conditioning any further would lead to conditioning upon the whole data, i.e. to a Bayesian solution. I also noted a curious lack of proper logical reasoning in the argument that, when

$$
f(z \mid \theta) \propto f(z \mid s) f(s \mid \theta),
$$

using the conditional ancillary distribution is enough, since "any departure from $f(z \mid s)$ implies that the overall model is false" (p.322), but not the reverse. Hence, a poor choice of $s$ may fail to detect a departure. (Besides the fact that fixed-dimension sufficient statistics $s$ do not exist outside exponential families.) Similarly, Spanos expands about the case of a minimal sufficient statistic that is independent from a maximal ancillary statistic, but such cases are quite rare and limited to exponential families [in the iid case]. Plus, the notion of conditional ancilarity is fraught with dangers, as exposed in Basu (1988). Still in the conditioning category, he also supports Mayo's argument against the likelihood principle being a consequence of the sufficiency and weak conditionality principles. However, he does not provide further evidence against Birnbaum's result, arguing rather in favour of a conditional frequentist inference I have nothing to complain about. (I fail to perceive the appeal of the Welch uniform example in terms of the likelihood principle.)

6. Conclusion. In a overall recap, let me restate that this note about Error and Inference is an extended reading note and is thus far from pretending at bringing a global and definitive Bayesian reply to the philosophical arguments raised in the volume. Once again, this broader perspective is partly provided by Gelman and Shalizi 
(2012). While the core goal is of "taking some crucial steps towards legitimating the philosophy of frequentist statistics" (p.328), the debate cannot escape veering at times towards a comparison with the Bayesian approach, hence generating the above comments of mine's. From a more global perspective, reading Error and Inference is a worthy and fruitful exercise for Bayesians and frequentists alike as most chapters at least should bring new food for their thoughts on hypothesis testing and model choice, since those inferential goals are still wide open to improvements and rebuttals within both approaches.

ACKNOWLEDGEMENTS. This research is supported partly by the Agence Nationale de la Recherche through the 2009-2012 grants Big MC and EMILE and partly by the Institut Universitaire de France (IUF). Comments from Andrew Gelman and discussions with Deborah Mayo are gratefully acknowledged.

\section{References.}

Aitkin, M. (2010). Statistical Inference: A Bayesian/Likelihood approach. CRC Press, Chapman \& Hall, New York.

BASU, D. (1988). Statistical Information and Likelihood: A Collection of Critical Essays by Dr. D. Basu. Springer-Verlag, New York.

Beaumont, M., Nielsen, R., Robert, C., Hey, J., Gaggiotti, O., Knowles, L., Estoup, A., Mahesh, P., Coranders, J., Hickerson, M., Sisson, S., Fagundes, N., Chikhi, L., Beerli, P., Vitalis, R., Cornuet, J.-M., HuelsenBeck, J., Foll, M., YAng, Z., Rousset, F., Balding, D. and Excoffier, L. (2010). In defense of model-based inference in phylogeography. Molecular Ecology, 19(3) 436-446.

Berger, J. (1985). Statistical Decision Theory and Bayesian Analysis. 2nd ed. Springer-Verlag, New York.

Berger, J. (2003). Could Fisher, Jeffreys and Neyman have agreed on testing? Statistical Science, 18 1-32.

Berger, J. and Bernardo, J. (1992). On the development of the reference prior method. In Bayesian Statistics 4 (J. Berger, J. Bernardo, A. Dawid and A. Smith, eds.). Oxford University Press, London, 35-49.

Berger, J., Ghosh, J. and Mukhopadhyay, N. (2003). Approximations and consistency of Bayes factors as model dimension grows. Journal of Statistical Planning and Inference, 112 241-258.

Berger, J. and Jefferys, W. (1992). Sharpening Ockham's razor on a Bayesian strop. American Statist., 80 64-72.

Berger, J. and Wolpert, R. (1988). The Likelihood Principle (2nd edition), vol. 9 of IMS Lecture Notes - Monograph Series. 2nd ed. IMS, Hayward.

Birnbaum, A. (1962). On the foundations of statistical inference. J. American Statist. Assoc., 57 269-306.

CARnap, R. (1952). Logical foundations of probability. University of Chicago Press, Chicago, IL, US.

DeGroot, M. (1970). Optimal Statistical Decisions. McGraw-Hill, New York.

Gelman, A. and Shalizi, C. R. (2012). Philosophy and the practice of Bayesian statistics. British Journal of Mathematical and Statistical Psychology. (To appear.).

Gigerenzer, G. (2002). Calculated Risks: How to Know When Numbers Deceive You. Simon and Schuster, New York.

Hartigan, J. A. (1983). Bayes Theory. Springer-Verlag, New York, New York.

Hwang, J., Casella, G., Robert, C., Wells, M. and Farrel, R. (1992). Estimation of accuracy in testing. Ann. Statist., 20 490-509. 
Jeffreys, H. (1939). Theory of Probability. 1st ed. The Clarendon Press, Oxford.

Kass, R. and Raftery, A. (1995). Bayes factors. J. American Statist. Assoc., 90 $773-795$.

Keynes, J. (1920). A Treatise on Probability. Macmillan and Co., London.

MacKay, D. J. C. (2002). Information Theory, Inference $\& 3$ Learning Algorithms. Cambridge University Press, Cambridge, UK.

Mayo, D. and Cox, D. (2006). Frequentist statistics as a theory of inductive inference. In Optimality: The Second Erich L. Lehmann Symposium (J. Rojo, ed.). Lecture Notes-Monograph Series, Institute of Mathematical Statistics, Beachwood, Ohio, USA, 77-97.

Mayo, D. G. (1996). Error and the Growth of Experimental Knowledge. University of Chicago Press.

Moreno, E., Girón, F. and Casella, G. (2010). Consistency of objective Bayes factors as the model dimension grows. Ann. Statist., 38 1937-1952.

O'Hagan, A. (1995). Fractional Bayes factors for model comparisons. J. Royal Statist. Society Series B, 57 99-138.

PÉREz, J. and Berger, J. (2002). Expected-posterior prior distributions for model selection. Biometrika, 89 491-512.

Robert, C. (2007). The Bayesian Choice. paperback ed. Springer-Verlag, New York.

Robert, C. (2010). An attempt at reading Keynes' treatise on probability. International Statistical Review. (To appear.).

Robert, C., Chopin, N. and Rousseau, J. (2009). Theory of Probability revisited (with discussion). Statist. Science, 24(2) 141-172 and 191-194.

Robert, C., Cornuet, J.-M., Marin, J.-M. and Pillai, N. (2011). Lack of confidence in ABC model choice. Proceedings of the National Academy of Sciences, 108(37) 15112-15117.

Schervish, M. (1995). Theory of Statistics. Springer-Verlag, New York.

Spiegelhalter, D. J., Best, N. G., Carlin, B. P. and van der Linde, A. (2002). Bayesian measures of model complexity and fit (with discussion). J. Royal Statist. Society Series B, 64 583-639.

Templeton, A. (2008). Statistical hypothesis testing in intraspecific phylogeography: nested clade phylogeographical analysis vs. approximate Bayesian computation. Molecular Ecology, 18(2) 319-331.

Templeton, A. (2010). Coherent and incoherent inference in phylogeography and human evolution. Proc. National Academy of Sciences, 107(14) 6376-6381.

von Mises, R. (1957). Probability, Statistics, and Truth. Dover, New York. 